\title{
Commissioning of the hybrid multibend achromat lattice at the European Synchrotron Radiation Facility
}

\author{
P. Raimondi, N. Carmignani®, L. R. Carver®, J. Chavanne, L. Farvacque $\odot$, G. Le Bec®, \\ D. Martin, S. M. Liuzzo®, T. Perron, and S. White® \\ ESRF, 38000 Grenoble, France
}

(Received 9 August 2021; accepted 18 October 2021; published 1 November 2021)

\begin{abstract}
The European Synchrotron Radiation Facility (ESRF) operates a $6 \mathrm{GeV}$ fourth generation light source, the ESRF-Extremely Brilliant Source (EBS). This storage ring is the first to implement the hybrid multibend achromat lattice (HMBA) that provides a reduction of the horizontal emittance of approximately a factor 30 with respect to the former double bend achromat structure. By combining several innovative concepts, the HMBA lattice allows the achievement of ultralow emittance while maintaining excellent lifetime and dynamic aperture performance. In this paper, beam commissioning experience and results of the new ESRF storage ring will be presented with an emphasis on the specific challenges relating to the HMBA lattice. The issues, methods, and concepts developed during the commissioning period and first months of operation are discussed.
\end{abstract}

DOI: 10.1103/PhysRevAccelBeams.24.110701

\section{INTRODUCTION}

The European Synchrotron Radiation Facility (ESRF) accelerator complex, shown in Fig. 1, consists of a $200 \mathrm{MeV}$ linac, a $6 \mathrm{GeV}$ booster synchrotron and a $6 \mathrm{GeV}$ storage ring light source. The linac and the storage ring are connected to the booster with two transfer lines, TL1 and TL2, respectively. X-ray sources and beam lines are located either in one of the 32 straight sections equipped with undulators (ID beam lines) or in the center of the arcs where the light is extracted from special bending magnets (BM beam lines).

Following the successful completion of the ESRF upgrade phase I [1], consisting of mostly infrastructures and beam lines improvements, the ESRF upgrade program phase II [2] aimed at replacing the existing double bend achromat (DBA) storage ring by an entirely new machine with a target horizontal emittance smaller than $150 \mathrm{pm} \mathrm{rad}$. There were two major constraints that strongly impacted the lattice design: maintaining the physical location of the source points while keeping the same infrastructure and tunnel and using the existing injectors chain with minimal adaptations. The project was organized in three main phases resulting in an interruption of users service mode (USM, operation with user experiments) of 20 months: 12 months were allocated to the dismantling of the old

Published by the American Physical Society under the terms of the Creative Commons Attribution 4.0 International license. Further distribution of this work must maintain attribution to the author(s) and the published article's title, journal citation, and DOI. storage ring and the installation of the new one, 3 months were dedicated to the machine commissioning, and 5 months to the beam lines commissioning. This report will focus on the machine commissioning phase.

\section{THE HYBRID MULTIBEND ACHROMAT LATTICE}

The multibend achromat (MBA) concept [3] involves increasing the number of dipoles and reducing the bending angle of each dipole and therefore reduces the equilibrium emittance. First introduced in 1995, this idea was demonstrated in 2015 for a $3 \mathrm{GeV}$ lattice when the MAX IV [4-6]

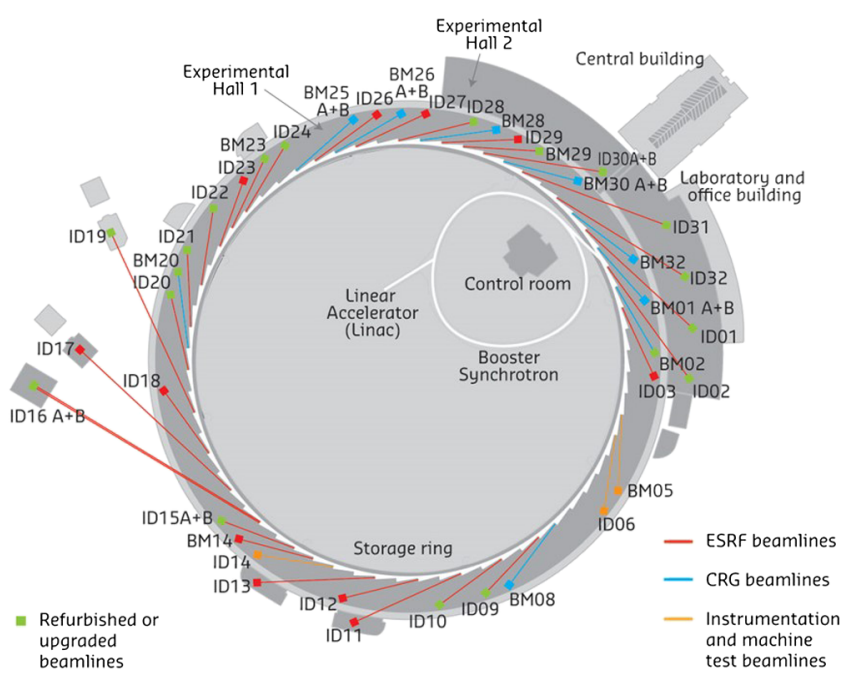

FIG. 1. ESRF accelerator complex and beam lines (image courtesy of the ESRF communication group). 
facility started operation. Adapting this concept for the ESRF $6 \mathrm{GeV}$ accelerator complex [7] was found impractical at the time when design studies began. The magnet strengths had to be increased beyond state of the art technology and the transverse and momentum acceptances were found too small to accommodate the standard off axis injection [8-10] and provide large beam lifetime [11,12]. More recent developments, such as swap-out and longitudinal on axis injection, now provide solutions to inject in very small acceptance $[13,14]$ but these were not considered during the design phase of the ESRF-EBS.

The technical solution proposed at ESRF, the HMBA lattice, introduces novel ingredients that allow a significant reduction in the magnet strengths while increasing the acceptance of the lattice and achieving ultralow emittances $[2,15]$. The main characteristics of the ESRF HMBA lattice design can be summarized as follows:

(i) Horizontal emittance of $133 \mathrm{pm} \mathrm{rad}(140 \mathrm{pm}$ rad with BM beam lines), (ii) Optimized sextupole layout and associated optics functions to reduce their strengths and allow shorter magnet lengths. This accommodates the increased number of dipoles while compensating for the natural chromaticity introduced by high gradient $\left(91 \mathrm{Tm}^{-1}\right)$ quadrupoles, and (iii) Intrinsic compensation of some nonlinear resonances to provide large transverse and momentum acceptances allowing for off axis injection with more than 9\% efficiency and beam lifetime on the order of $20 \mathrm{~h}$.

In addition, the overall power consumption is reduced compared to the previous DBA lattice thanks to the smaller radiated power $U_{0}$ and to the usage of permanent magnet technology for some dipoles.

The unit cell of the ESRF HMBA lattice is shown in Fig. 2. It starts at the center of the $5.3 \mathrm{~m}$ insertion devices (ID) straight section followed by a matching doublet, a chromatic correction section (CCS) surrounded by two longitudinal gradient dipoles (DL) and a central section with strong focusing quadrupoles (QF) and combined function defocusing dipole-quadrupole (DQ) magnets. Unless there is a BM beam line, the cell is symmetric around the central DQ magnet. All the magnets are powered individually and there are ten beam position monitors (BPM) per cell to allow for precise orbit, coupling and optics corrections and control. The correctors (SH magnets) and sextupoles (focusing SF and defocusing SD) are all able to produce horizontal and vertical dipole fields as well as a skew quadrupole field. In addition, the DQ magnets are equipped with a correction coil that provides independent control of the bending angle and field gradient with a range of approximately $1 \%$. Finally, the SH magnets are able to produce fast varying horizontal and vertical dipole fields with bandwidths of approximately $150 \mathrm{~Hz}$ in the horizontal plane and $300 \mathrm{~Hz}$ in the vertical. These bandwidths were estimated from measurements of the beam response to a sinusoidal excitation and are limited by the vacuum chamber.

The ESRF-EBS main ring parameters and comparison with the previous ESRF DBA lattice are summarized in Table I. The dipole layout is defined such as to leave space for the two CCS and optimize the $\beta$-functions and dispersion at the sextupoles, allowing for shorter, weaker magnet design. The longitudinal gradient permanent dipole magnets are used to further increase the dispersion and reduce the horizontal emittance [16,17]. The central part of the cell is designed to optimize the space usage and increase the horizontal damping partition number $J_{x}\left(\epsilon_{h} \propto J_{x}^{-1}\right)$. An odd multiple of $\pi$ phase advance, or $-\mathcal{I}$ transform [18], between focusing sextupoles is imposed to partially cancel nonlinear resonances [19-21]. With all these ingredients integrated in the lattice, it was possible to increase the filling factor, $L_{\mathrm{dip}} / L_{\text {total }}$, of the machine and therefore reduce the equilibrium emittance without compromising the machine performance in terms of lifetime and acceptance. The sextupole strengths required for the HMBA lattice of the ESRF $6 \mathrm{GeV}$ storage ring are fully compatible with present technological capabilities [22-26].

While the ESRF DBA lattice was alternating highand low-horizontal $\beta$ straight sections, the HMBA lattice features 31 identical straight sections hosting either ID or rf

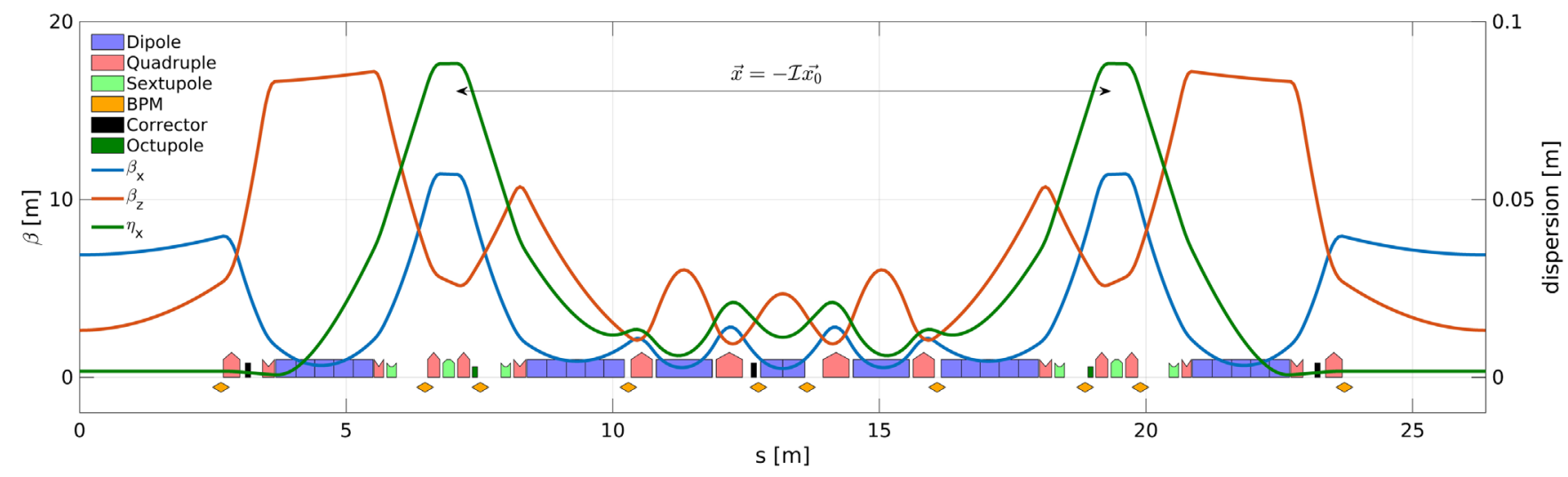

FIG. 2. Lattice functions and magnets layout for the HMBA SR standard cell with two symmetric CCS. Horizontal and vertical steerers and skew quadrupoles are located in each corrector and sextupole depicted in the layout. 
TABLE I. Main parameters of the ESRF DBA and HMBA lattices. The multipole strengths $K_{\mathrm{n}}$ include the factor $1 / n$ !

\begin{tabular}{|c|c|c|c|}
\hline & Units & DBA & HMBA \\
\hline Energy & $\mathrm{GeV}$ & 6 & 6 \\
\hline Circumference & $\mathrm{m}$ & 844.44 & 844 \\
\hline Number of cells & & 32 & 32 \\
\hline Dipole filling factor & $\%$ & 18 & 38 \\
\hline $\begin{array}{l}\text { Horizontal } \beta @ \text { insertion } \\
\text { devices (ID) }\end{array}$ & $\mathrm{m}$ & $37.6,0.4$ & 6.9 \\
\hline Vertical $\beta @$ ID & $\mathrm{m}$ & 3.0 & 2.7 \\
\hline Horizontal emittance & pm rad & 3985 & 133 \\
\hline Vertical emittance & pm rad & 4 & 1 \\
\hline Momentum spread & $\%$ & 0.106 & 0.094 \\
\hline Bunch length (@0mA) & $\mathrm{mm}$ & 3.43 & 2.9 \\
\hline Beam current & $\mathrm{mA}$ & 200 & 200 \\
\hline $\begin{array}{l}\text { Horizontal damping } \\
\text { partition number }\end{array}$ & & 1.00 & 1.51 \\
\hline Energy loss/turn & $\mathrm{MeV} /$ turn & 4.88 & 2.56 \\
\hline Maximum $K_{1}$ (quadrupole) & $\mathrm{Tm}^{-1}$ & 16 & 91 \\
\hline Maximum $K_{2}$ (sextupole) & $\mathrm{Tm}^{-2}$ & 222 & 1720 \\
\hline Maximum $K_{3}$ (octupole) & $\mathrm{Tm}^{-3}$ & 0 & 36025 \\
\hline Magnets/cell & & 19 & 32 \\
\hline
\end{tabular}

cavities and one straight section dedicated to injection. At the injection point, the horizontal $\beta$-function is increased to $18.6 \mathrm{~m}$ to enlarge the transverse acceptance and allow for high efficiency off axis injection. This breaks the 32-fold symmetry, however, the lifetime performance is restored to approximately $20 \mathrm{~h}$ (assuming a lattice with errors and approximately $0.2 \mathrm{~mA}$ per bunch, $5 \mathrm{pmrad}$ vertical emittance and $3.5 \mathrm{~mm}$ bunch length at this current) with appropriate sextupole optimizations [27,28]. The vertical $\beta$-function of the ID straight sections was determined based on a configuration with two $2 \mathrm{~m}$-long undulators. However, lifetime and dynamic aperture (DA) constraints did not allow to maintain the horizontal $\beta$-function to the optimal value for ID beam lines. The optics functions at BM beam lines are constrained by lattice considerations. The BM beam lines used to extract light from the dipoles of the DBA lattice. For the HMBA lattice, dedicated sources were designed to compensate the reduction of bending angle and maintain photon beam characteristics [29]. Three types of source are used, a single dipole or short bend (SB), a 2-dipole wiggler (2PW), and a 3-dipole wiggler (3PW) [30]. The integration of the $\mathrm{SB}$ and $2 \mathrm{PW}$ sources require a modification of the alignment of neighboring magnets in order to compensate for the additional angle or offset, respectively. The $3 \mathrm{PW}$ is self-compensated and does not require any modification of the lattice. With all the BM beam lines integrated in the lattice, the horizontal emittance increases by $6 \mathrm{pm}$ rad to reach approximately $140 \mathrm{pm}$ rad [29].

\section{BEAM COMMISSIONING EXPERIENCE}

This section provides an overview of the storage ring beam commissioning. A chronological timeline with the commissioning highlights is given for reference in Fig. 3. However, only few selected topics will be discussed in this paper. It should be noted, that the success of the beam commissioning relied heavily on the excellent preparation and readiness of all critical subsystems. A storage ring control system simulator was implemented to allow the development and test of most of the controls and numerical tools in the control room environment well before the start of the commissioning. Based on the solid experience acquired on the previous machine and clever adaptation of many systems, the diagnostics, power supplies (PS), rf systems, magnets, and their controls provided excellent performance from day one and throughout the whole commissioning period.

\section{A. Injectors commissioning}

In order to comply with a very tight commissioning schedule and to mitigate the overall risks for the project completion, it was decided to minimize and bring forward modifications on the injectors chain. In this spirit, a complete refurbishment of the linac, the booster power supply and rf systems, and the renewal of the global timing system were performed prior to the dismantling of the old storage ring and the standard off axis injection was maintained. However, necessary adaptations of the booster and transfer lines took place [2]: (i) Reduction of the booster circumference to match the storage ring length, (ii) Modified layout of the transfer lines to adapt to the new injection and extraction points, and (iii) Adaptation of the injection and extraction systems.

Although the reduction of the booster circumference represented a major intervention, it was completed according to schedule and the restart of the injectors took only a few days. The booster was foreseen to operate off energy with higher integer tunes, i.e., new optics, to reduce the horizontal emittance and improve the transfer efficiency [31]. This new

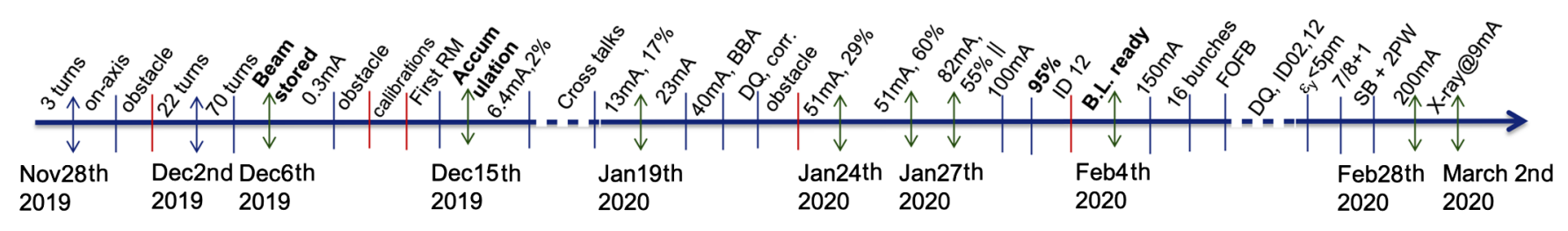

FIG. 3. EBS commissioning chronological timeline. 
optics was not immediately implemented and is still under development. This was compensated by the implementation of an emittance exchange scheme at the booster extraction that provided a substantial horizontal beam size reduction and an increase in injection efficiency of 5-10\% [32].

\section{B. First turns and accumulation}

Tests and validations of first turns steering performed in the previous machine combined with simulation for the ESRF-EBS indicated that it was possible to establish the closed orbit starting from off axis injection with all magnets at their design strengths [33]. This strategy was quickly revised as major difficulties were faced during the first weeks of commissioning due to unexpected reductions of both mechanical and DAs which required the minimization of injection oscillations. It was later possible to identify and associate these reductions to either physical obstacles inside the vacuum chambers or unforeseen large magnetic errors due to cross talk between magnets [34] and wrongly assigned calibration factors [35].

As seen in Fig. 3, a total of three obstacles were found and removed during the first months of commissioning. The first two obstacles were found in the first month of commissioning and consisted on a piece of heating wire located in a straight section chamber and a shielded bellow chamber mounted upside-down. The third obstacle was found later, after 2 months of commissioning, and consisted in a piece of aluminium foil located at the center of an arc (DQ chamber). This last obstacle was located further away from the beam axis and its impact on machine performance became visible only with well tuned orbit and optics. As a consequence, the storage ring had to be operated for a long period on a dispersive orbit, with a frequency shift of $300 \mathrm{~Hz}$, and at times with nonzero closed orbit or local orbit bumps until this last obstacle was finally found and removed. All obstacles were diagnosed and located using beam loss detectors (BLD), beam position monitors (BPM) turn-by-turn data [36], orbit bump scans, and radiation survey inside the tunnel for precise localization.

Figure 4 shows an example of the combined usage of the BLD system and a closed orbit bump to detect the last obstacle. In this case, a $\pi$ bump using the two focusing sextupoles on either side of the $-\mathcal{I}$ transform was used. Obstacles in straight sections were identified using four steerers parallel bumps. Once all the obstacles were removed and the magnetic model corrected, the machine performance steadily improved throughout the commissioning period without major difficulties.

First turns could nevertheless be established using off axis injection with a $15 \mathrm{~mm}$ bump amplitude leading to a theoretical separation of $5 \mathrm{~mm}$ between the stored and injected beams at the injection point. 2.5 turns were achieved by simply adjusting the injectors and incoming trajectory without any need of correction in the storage ring, i.e., all correctors off. This was unexpected as
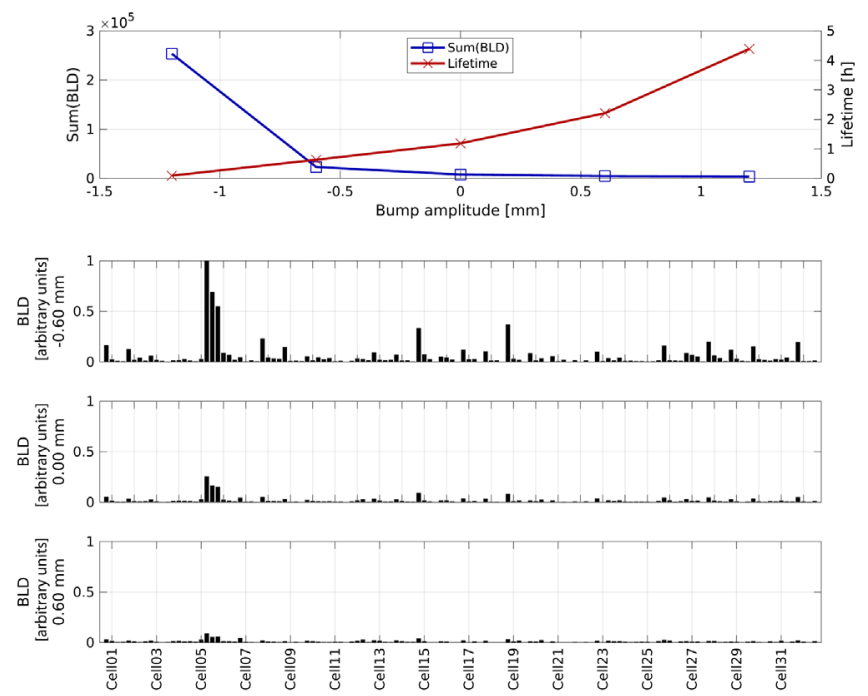

FIG. 4. Obstacle detection in the middle of the arc using a local bump. The total losses and lifetime are shown on the top plot and the loss pattern for three bump amplitudes are shown at the bottom. The asymmetry observed is a clear signature of an obstacle.

simulation using alignment tolerances predicted that only one third of a turn would be achieved without steering and gave a first indication on the excellent storage ring alignment achieved during the installation phase.

The first turns trajectory measurements are shown in Fig. 5. The horizontal and vertical trajectories are shown together with the BPM sum signals, that are proportional to the beam current. A polynomial fit algorithm [37] of the expected BPM nonlinearity at large amplitude was used in order to provide accurate measurement of the injected beam trajectory. The horizontal injection oscillations of a few millimeters are consistent with a $15 \mathrm{~mm}$ bump amplitude. A clear drop in current (proportional to the BPM sum signal) at approximately 1.5 turns (BPM sample 510, 320 BPMs per turn) is observed. This was the first clear indication of a physical obstacle later found in straight section 23. Due to the significantly reduced acceptance, it was not possible to proceed further using off axis injection. A combination of a static closed orbit bump and increased injection kickers

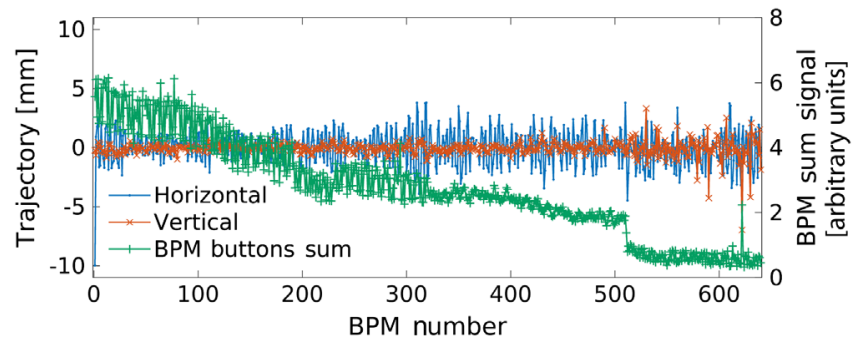

FIG. 5. First turns trajectory in the storage ring with off axis injection. Blue is horizontal and red vertical. Green is BPM buttons sum signal. 
strengths was used to establish pseudo on axis injection (a few millimeters residual was measured). This allowed to progress with beam threading and establish multiple turns. The static bump introduced additional $\beta$-beating $(\sim 5 \%)$ and could only be removed one month after the start of commissioning once the acceptance was sufficiently enlarged.

Multiturn beam threading was performed using a semiautomated trajectory correction on the first turns. The correction algorithm is based on singular value decomposition and described in Ref. [33]. The initial positions and angles measured using the first two BPMs were optimized using the two injection septa and the last two vertical correctors of the TL2 transfer line. In order to exclude BPM data correlated with low-beam current, a predefined validation threshold on the BPM sum signal was used. Below this threshold, the data point is ignored and the acquisition buffer is truncated following three consecutive readings below this threshold. The horizontal and vertical planes were corrected individually. The sextupoles and octupoles were adjusted by families (two families of SD and one family of SF and octupoles) in an iterative process to optimize the number of survival turns. After optimization, the sextupoles were reduced to approximately $60 \%$ of their nominal operating point, the octupoles were turned off and the horizontal and vertical tunes were shifted by +0.5 and -1.4 units, respectively, to obtain few tens of turns with an rms oscillation amplitude of approximately $750 \mu \mathrm{m}$. These significant deviations from the theoretical model indicated potential large errors in the magnetic description of the lattice.

In theory, 70 turns can be performed in the storage ring with rf systems off before the beam is lost. However, due to the large deviations with respect to the theoretical model, sextupole optimization proved to be essential to achieve the 70 turns required to switch on the rf systems and allow for capture. This is shown in Fig. 6 where the survival rate is shown as a function of turns. The settings optimized for first turns were determined experimentally and feature a flattop at low-turn numbers. On the other hand, the settings optimized for rf capture were defined based on tracking simulations of survival rate in realistic conditions and show a plateau at large turn numbers.

Beam accumulation is not possible with on axis injection. The injection kickers were therefore optimized to share oscillations between the stored and injected beams and to allow injection in the reduced acceptance. A maximum total current of $6 \mathrm{~mA}$ was accumulated with an injection efficiency of $0.8 \%$ during the first 3 weeks of commissioning. Following this initial commissioning period, the cross talks were included in the magnetic model and calibrations factors were properly assigned. It was then possible to perform closed orbit correction and optics tuning to proceed with the ramp in current and vacuum conditioning to deliver operating conditions compatible with the start of beam lines commissioning.

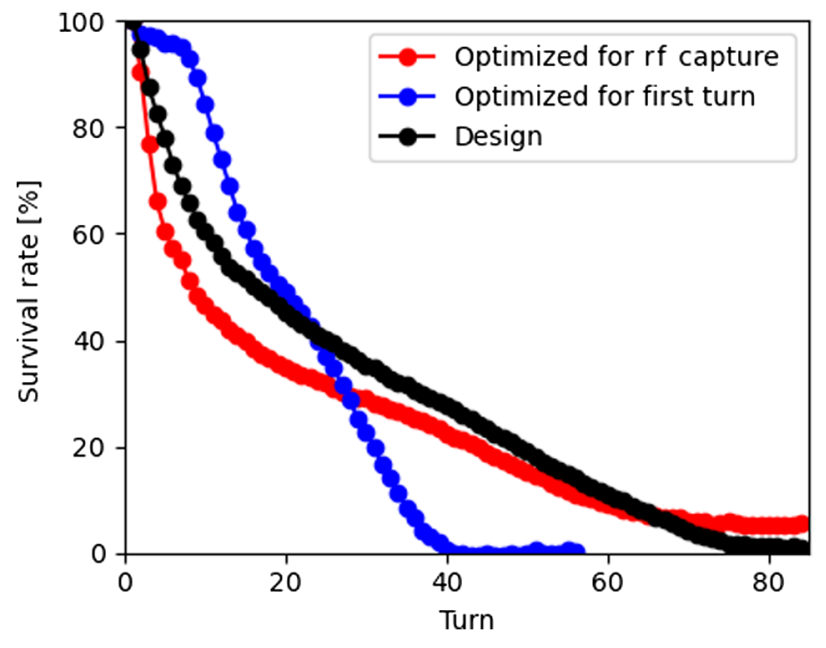

FIG. 6. Survival rate derived from the BPM turn-by-turn sum signal for different sextupole settings used during the commissioning.

\section{CLOSED ORBIT AND LINEAR OPTICS CORRECTIONS}

As discussed in the first section of this paper, the HMBA lattice is the main and essential ingredient for the ESRFEBS project. Excellent optics control and characterization are therefore essential to fulfill the design constraints and deliver the expected performance. This section summarizes the methods and actions taken during the commissioning period to first minimize the closed orbit, an essential step to achieve precise optics control, and then correct the lattice toward the theoretical model including the integration of the BM beam line sources.

\section{A. Closed orbit steering}

The initial rms closed orbit of several hundred microns was in large part due to uncorrected offsets, static injection bump, and a conservative approach to orbit correction motivated by the presence of multiple obstacles. This large initial value could be reduced down to approximately $50 \mu \mathrm{m} \mathrm{rms}$ in both planes following a number of corrective measures.

Closed orbit correction relies on the accuracy and reliability of the BPM system. BPM data can suffer various uncertainties, or offsets, related to mechanical alignment or electronic errors. These need to be measured and compensated to achieve the desired performance. Before the start of beam commissioning mechanical offsets were applied based on the alignment survey and large electronic offsets were corrected. Large offsets however remained and beambased alignment (BBA) proved to be an essential step. The BPM offset is measured with respect to the nearest quadrupole magnetic center [38]. A total of 320 BPMs were measured and corrected in approximately $40 \mathrm{~h}$. Initially, each plane was measured separately to ensure safe manipulation of the beam. However now that the orbit is much 

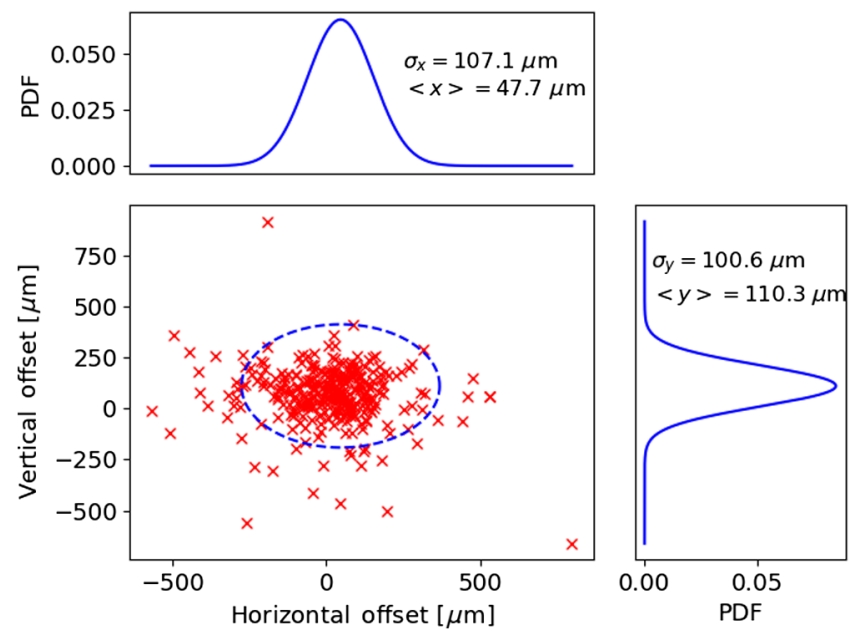

FIG. 7. Measured BPM offsets and probability density functions (PDF) in the horizontal and vertical planes. The blue dashed line is placed at $3 \sigma$.

better corrected, both horizontal and vertical planes can be scanned simultaneously which reduces the time taken to approximately $16 \mathrm{~h}$. Fast BBA described in Ref. [39] is under development. Figure 7 shows the result of the BBA measurement campaign, rms offsets of approximately $100 \mu \mathrm{m}$ were found in both planes. These values are lower than initial estimates that assumed a worst case scenario of $500 \mu \mathrm{m} \mathrm{rms}$ offsets. The nonzero average values are attributed to systematic errors on the mechanical offsets measurements that were applied before the start of the commissioning. BBA had an immediate impact on rms orbit, orbit corrector strengths, and optics corrections.

The orbit correction is performed using standard singular value decomposition methods as presented in Ref. [18]. Simulation using design alignment tolerances predicted that a maximum of approximately 100 singular vectors could be used over a total of 288 available. Exceeding this value would result in correctors running out of strength. Before the start of commissioning, the corrector PS capabilities were increased to partially mitigate this limitation. In reality, the better than expected alignment of the storage ring allowed the operation of the machine with corrector strengths well within PS limits and the exploration of the potential to increase the number of singular vectors and better correct the closed orbit.

Simulations were used to determine the optimum number of singular vectors. A set of lattices with random quadrupole errors $\quad\left(\sqrt{\left\langle\Delta x^{2}\right\rangle}=60 \mu \mathrm{m}, \quad \sqrt{\left\langle\Delta y^{2}\right\rangle}=40 \mu \mathrm{mrms}\right.$, corresponding to our best knowledge of alignment errors at the time and later confirmed by simulations as discussed in Sec. IV D) was generated and closed orbit corrections were performed with increasing number of singular vectors. Figure 8 shows the results of these simulations. Correcting with 162 singular vectors in both planes showed a net improvement in DA and $\beta$-beating. These values were then

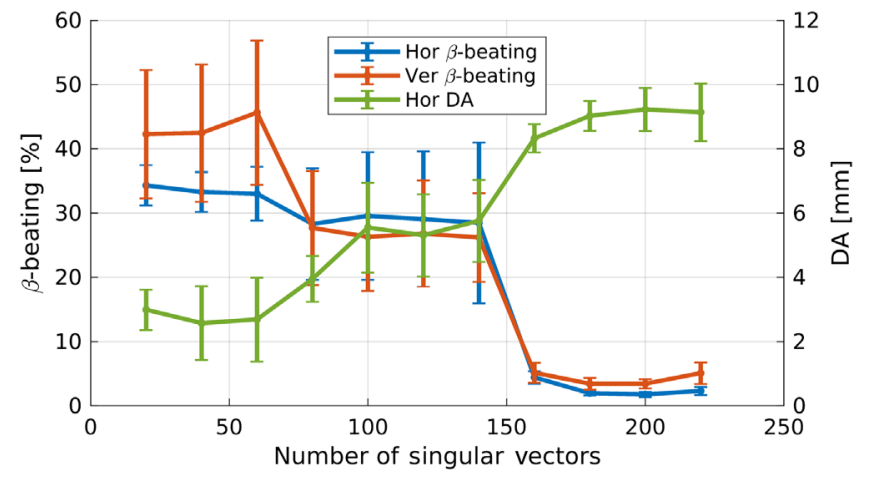

FIG. 8. Simulated evolution of the dynamic aperture and $\beta$-beating as a function of the number of singular vectors used for closed orbit correction in lattices with quadrupole alignment errors of $\sqrt{\left\langle\Delta x^{2}\right\rangle}=60 \mu \mathrm{m}, \sqrt{\left\langle\Delta y^{2}\right\rangle}=40 \mu \mathrm{m}$ using ten seeds of errors.

used in operation. The corrector strengths remained well within limits and this resulted in improved closed orbit and better injection efficiency. Although simulations indicate a potential improvement in DA, pushing further the number of singular vectors lead to a strong increase in corrector strengths without obvious gain in performance.

The decomposition in singular vectors of the horizontal correctors pattern present in the storage ring was showing two interesting features as seen on the top plot of Fig. 9. First, singular vectors above 162 were present. This was unexpected since all vectors above 162 were excluded from
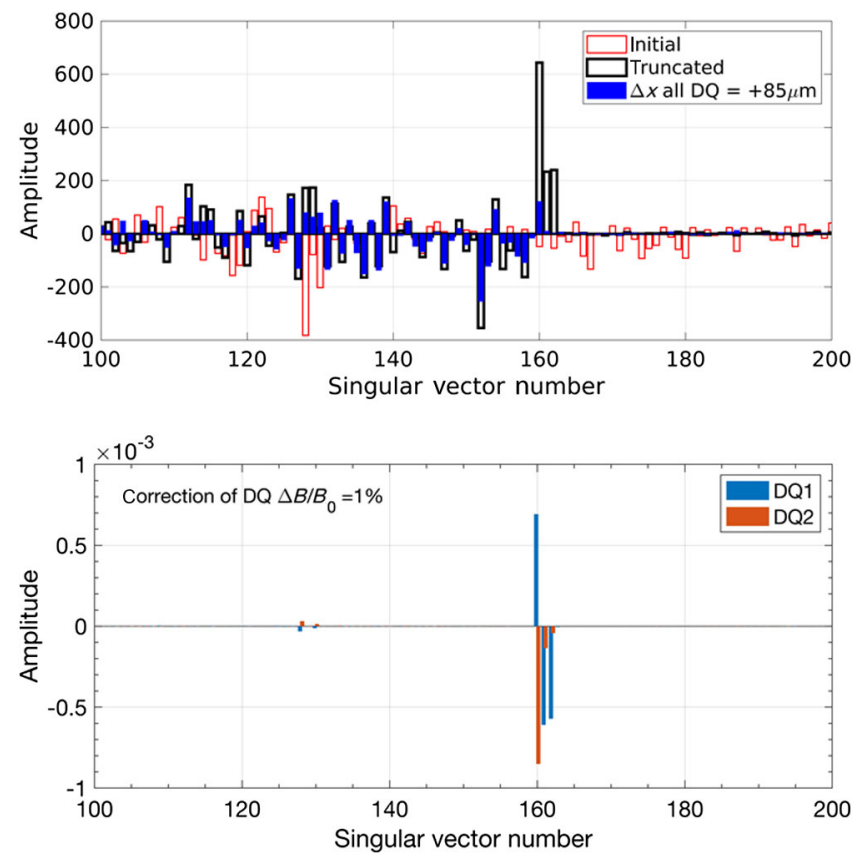

FIG. 9. Top: Horizontal correctors decompositions following corrective actions on the closed orbit. Bottom: Decomposition of the steering pattern required to fix a field error of $1 \%$ in each DQ magnet. 
the correction and most probably the result of several correctors calibration issues and leftovers from bumps used during the commissioning. Second, very strong singular values 160,161 , and 162 were present. The first observation was solved by truncating of all unwanted singular values, above 162 as seen on the black curve. This truncation is now part of the automated orbit correction loop as it was found to be crucial for the correct interaction between fast and slow orbit correction. The fast correction is acting on a subset of BPMs and correctors of the slow correction that is using the full complement and was therefore polluting the correctors pattern with high order vectors and therefore artificially increasing the corrector strengths.

The detailed analysis of singular vector 162 is of particular interest as it turned out to reflect an issue with the magnetic description of the lattice and was essential to diagnose a missing bending angle (or misalignment) in the DQ magnets. In the bottom plot of Fig. 9, we can see that the contribution of field errors in the DQ magnets to the singular vectors are all concentrated around vector 162 . In fact, it was possible to fit the measured vectors 160,161 , and 162 using strictly DQ alignment errors. Although this provided a strong indication that realigning the DQs was required this hypothesis was first validated by changing the magnet fields. At this stage of the commissioning, the horizontal trim coils of the DQ magnets were not available and the correction angle was applied by changing the DQ gradients. With this correction applied, the rms horizontal corrector strengths was reduced from $87 \mu \mathrm{rad}$ to $50 \mu \mathrm{rad}$ while maintaining an rms closed orbit of $60 \mu \mathrm{m} \mathrm{rms}$ in both planes. Simultaneously, the quadrupole corrections applied to adjust the tune had been reduced, potentially indicating an overall operating point closer to the model. Following these observations, it was decided to displace all DQ magnets horizontally by $85 \mu \mathrm{m}$ to recover their nominal gradient. These movements proved to be insufficient as they allowed to cancel only half of the correction. However, the remaining DQ gradient errors had no impact on the lattice performance and were integrated in the model. It was later discovered that the lattice model was based on the DQ mechanical lengths rather than their magnetic lengths, introducing a dipole field error, and that one family of DQ magnets suffered an alignment reference issue that lead to an error of $121 \mu \mathrm{m}$. These validated the intervention and the final result is shown on the blue curve in Fig. 9 for which the vectors 160,161 , and 162 are strongly reduced.

The DQ trim coils were finally made available and integrated in the correction algorithm. The number of singular values was increased from 162 to 200 and the closed orbit without bumps was reduced to approximately $50 \mu \mathrm{m}$ in both planes. However, several DQ magnets were showing large correction values for the horizontal steering (above $200 \mu \mathrm{rad}$ ). This was interpreted as a sign of an incorrect field or alignment. Assuming their mechanical and alignment tolerances were fulfilled, these errors were too large to be explained by errors in the DQ magnets only. A pragmatic approach was therefore adopted: (i) in case the DQ had to moved, to install a BM source [29] for instance, correct the error with the DQ magnets and (ii) otherwise use the adjacent quadrupoles with stronger gradient to minimize the movements amplitudes. After the correction is applied and the orbit is steered back to its nominal value, the DQ magnets are frozen and excluded from the orbit correction loop.

The closed orbit is maintained stable over time using two distinct feedback loops. The slow orbit correction loop that corrects the orbit every minute using all available steerers and BPMs and a fast orbit correction loop (Fast Orbit FeedBack, FOFB) using a subset of steerers capable of generating fast varying fields (SH magnets only) and BPMs with a $10 \mathrm{kHz}$ output (BPM number 1,2,4,7,9, and 10 of each cell). Most of the controls and electronics of these systems were kept from the previous machine and the possibility to run them in parallel had been validated before the long shutdown [40]. The recommissioning of these systems was therefore not an issue and it was possible to restart them almost immediately.

\section{B. Linear optics corrections}

Manual optics adjustments and corrections were performed very early in the commissioning while trying to establish stored beam. The first observation of strong optics distortions were in fact estimated from first turn trajectory data that gave a vertical tune error of -1.4 units with respect to the model ( $25.9 \pm 0.3$ instead of 27.34). The first turn data featured a continuous phase drift with respect to the model expectations that indicated small distributed errors rather than a strong localized imperfection. Optics measurements based on orbit response matrix (ORM) acquisition confirmed this hypothesis: rms $\beta$-beating of $20 \%$ and $34 \%$ and rms dispersion mismatch of 23.6 and $4.3 \mathrm{~mm}$ in the horizontal and vertical planes, respectively, were derived from these measurements as shown in Fig. 10.

Such large optics errors were not expected and the resulting corrections to fit the measurements were found to be unrealistic. These observations lead to a complete reassessment of the magnetic model of the lattice that shed light on two major issues. First, due the compactness of the lattice in the longitudinal direction, significant cross talks between adjacent magnets exist that alter its magnetic layout. This had only been considered for the interaction between the fringe fields of the DLs with the neighboring quadrupoles, but in fact it was present for all neighbouring magnets with each other. Second, calibration factors based on magnetic measurements performed prior to the installation were wrongly assigned or suffered from measurements errors resulting in erroneous current to field conversion. The source of the measurement errors was 

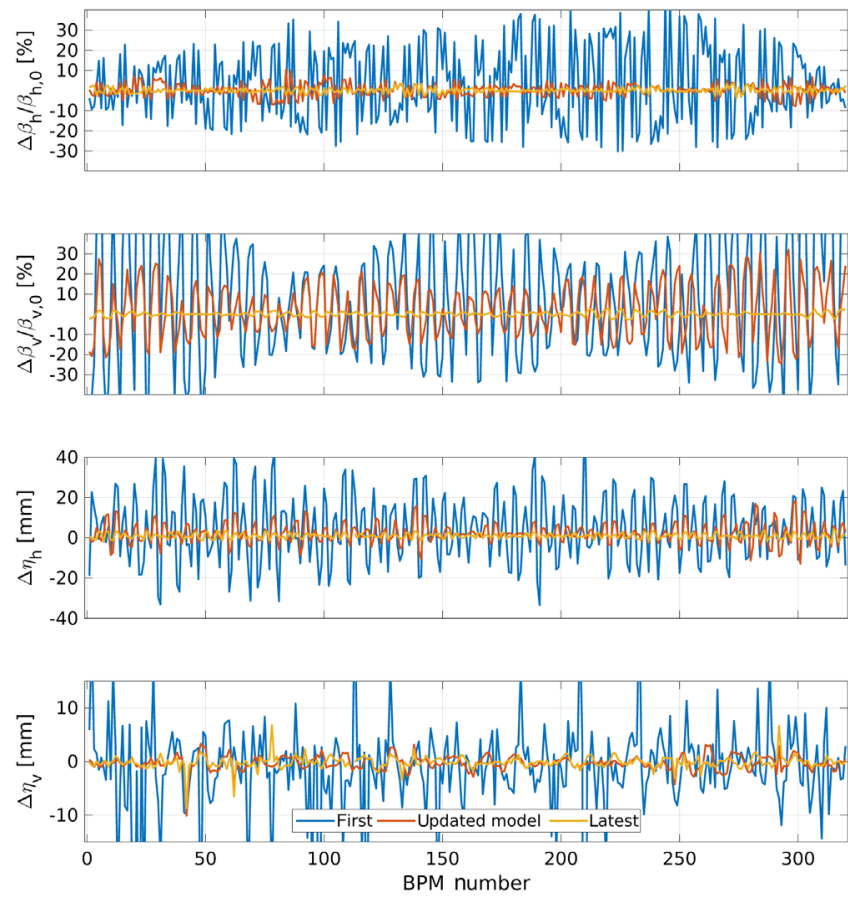

FIG. 10. Dispersion and optics measurements. (blue) First measured 9th December. (red) After introduction of cross talks, calibration, BBA, steering, no optics correction. (yellow) After optics correction.

due to either wrong cycling or current setting during the calibration curve characterization [41].

The computation of magnetic cross talks is described in details in Ref. [34]. These errors were computed in simulations and then validated by magnetic measurements using the stretched wire method [42]. Figure 11 shows an example of the gradient distribution in the longitudinal direction in the presence of a DL magnet (permanent dipole) and a quadrupole. The blue line represents the additional gradient introduced by the fringe field of the quadrupole interacting with the DL magnet that leads to a reduction of approximately $1 \%$ of the total integrated

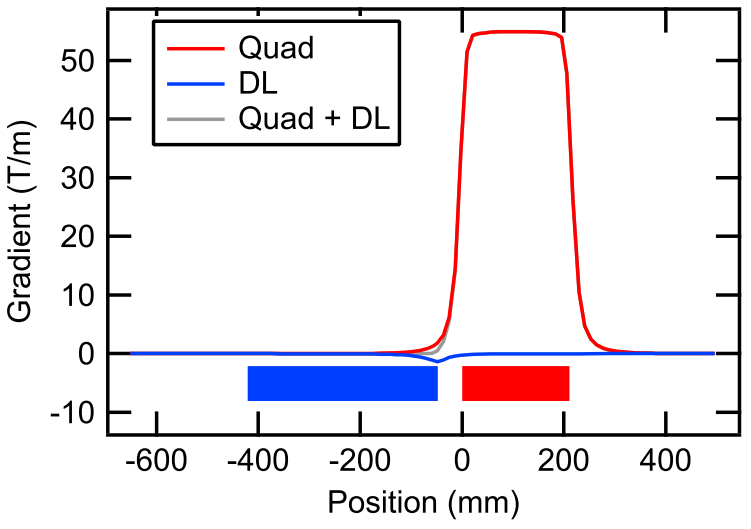

FIG. 11. Example of quadrupole gradient cross talk effect between a DL module in blue and a QD magnet in red. gradient in this configuration. Similarly, the dipole field of the module closest to the quadrupole is reduced. While the field reduction of the DL magnet was anticipated and compensated at the design phase, the gradient error was initially not integrated in the model leading to significant optics errors. Magnetic cross talks are also relevant in other configurations such as sextupole-quadrupole or octupolequadrupole for which the resulting field error is as high as $1.7 \%$. The most relevant configurations are listed in Table II. The large errors on the defocusing quadrupoles (QD) close to longitudinal dipoles (DL) contribute to the large vertical tune offset while the errors in the QF located in a dispersive region, contribute mainly to the dispersion beating. The predicted impact of these gradient errors on the vertical tune corresponds to a deviation of -1.3 units in the vertical plane that is comparable to the measured initial value of -1.4 units. The cross talks were accounted for by modifying the calibration scale factors of the magnets and by inserting thin elements where appropriate and rematching the lattice to the design optics functions. On the example shown in Fig. 11, the main quadrupole gradient in red is rescaled and a thin quadrupole lens is added at the edge of the dipole magnet in blue where the gradient error reaches a maximum.

On top of the inexact magnetic description of the lattice, additional calibration errors were found that contributed to the initial large optics errors with multiple sources such as wrongly assigned scale factor or magnet serial number, controls issues or wrong cycling during magnetic measurements that accumulated to errors as large as $1 \%$.

TABLE II. Simulated quadrupole gradient cross talks for the standard cell. All percentages relate to the total strength of the main magnet of the central column. Body indicates that the gradient error is distributed across the full magnet length while edge indicates that the field error is localized at its edge as shown in Fig. 11.

\begin{tabular}{lcc}
\hline \hline Left magnet & Main magnet & Right magnet \\
\hline & QD2 & DL1A \\
& $-0.088 \%$ body & $-0.792 \%$ edge \\
DL1 & QD3 & SD1 \\
$-0.93 \%$ edge & $-0.141 \%$ body & $-0.342 \%$ edge \\
& QF4 & SF2 \\
& $-0.03 \%$ body & $-0.27 \%$ edge \\
SF2 & QF4 & OD \\
$-0.27 \%$ edge & $-0.03 \%$ body & $1.7 \%$ body \\
SD1 & QD5 & DL2 \\
$-0.274 \%$ edge & $-0.056 \%$ body & $-0.84 \%$ edge \\
DL2 & QF6 & DQ1B \\
$-0.02 \%$ edge & $-0.082 \%$ body & $0.04 \%$ body \\
DQ1B & QF8 & \\
$0.042 \%$ body & $-0.061 \%$ body & \\
\hline \hline
\end{tabular}


Although the control system simulator was an excellent tool to identify and track these errors while commissioning activities could proceed, it would have been useful to perform more consistent and systematic checks before the start of commissioning and potentially avoid these issues. Optics measurements performed after the cross talks were integrated in the model and the calibration factors were properly assigned are shown on the red curves in Fig. 10. A clear improvement with respect to the initial situation is observed. Figure 12 shows the result of the last iteration on the magnetic model integrating all the above mentioned corrections and further corrections on the DQ magnets. The residual correction strengths are significantly reduced for most magnet families. Lifetime and DA performance were found unchanged with this new optics.

With these large errors removed, accurate optics corrections were made possible; however, several difficulties were faced before a clear procedure could be established. The ORM method is used at ESRF for optics and coupling corrections [43]. This method consists of measuring the closed orbit response to a dipole field change. Extensive studies, benchmarking, and validation of the method as well as comparative measurements with other methods were performed on the old ESRF ring $[44,45]$. Two types of dipole correctors can be used to perform such measurement: the correction coils of the sextupoles or the $\mathrm{SH}$ magnets. All correctors suffer from large hysteresis effects resulting in significant systematic error in the ORM. As a result, measuring a single corrector response would lead to a residual orbit $\Delta x$ of approximately $10 \mu \mathrm{m} \mathrm{rms}$. This effect was accumulating at each corrector response measurement eventually leading to a final closed orbit after measurement of approximately $100 \mu \mathrm{m} \mathrm{rms}$ and introducing artificial systematic errors due to feed-down effects in sextupoles. To overcome this issue single corrector orbit correction after each individual corrector response measurement was implemented and allowed to cancel the errors at the source. Large correctors scaling errors, however, remain on the SH magnets due to hysteresis effects when operating close to

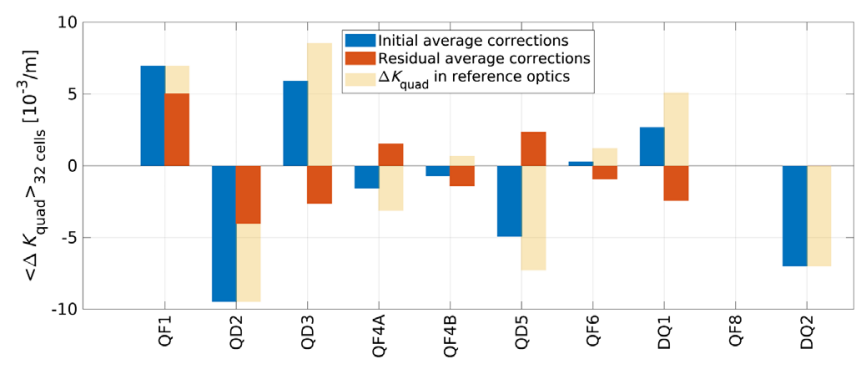

FIG. 12. Final iteration on optics model adaptation. Each bar represents an average by family. The blue bars are the measured correction strength before the DQ corrections. The yellow bars are the gradient changes introduced by the new reference optics with DQ corrections and the red bars are the residual corrections (initial-new reference). zero current. These effects are very complex to model and could not be integrated in the calibration curves. Providing it had no impact on the final result, the SH magnets were excluded from the ORM measurements and only sextupole corrector coils were used. Using two correctors per cell (64 total) was found to be a good compromise between measurement duration and precision.

Optics corrections gradually improved together with our understanding of the machine and the removal of strong localized errors. This progression is shown on Fig. 10. The first optics measurements in blue featured rms $\beta$-beating of $\Delta \beta / \beta_{h}>19.5 \%$ and $\Delta \beta / \beta_{v}>34 \%$ and $\mathrm{rms}$ dispersion beating of $\Delta \eta_{h} \simeq 23.6 \mathrm{~mm}$ and $\Delta \eta_{v} \simeq 4.3 \mathrm{~mm}$ in the horizontal and vertical planes, respectively. At this point in the commissioning, the procedures and fitting algorithms were not perfectly well established. These estimates should therefore be considered as a lower limit as they may suffer from large uncertainties. Following the correction of calibration factors and the integration of magnetic cross talks and DQ corrections in the theoretical model, measurements were performed on the bare lattice (without corrections) to estimate what would have been the ideal starting point. This measurement corresponds to the red curves where rms dispersion and $\beta$-beating of $\Delta \beta / \beta_{h} \simeq$ $3.4 \%$ and $\Delta \beta / \beta_{v} \simeq 13.3 \%$ and $\Delta \eta_{h} \simeq 5.1 \mathrm{~mm}$ and $\Delta \eta_{v} \simeq$ $1.3 \mathrm{~mm}$ are observed. In these conditions, the injection efficiency (IE) from the TL2 transfer line to the storage ring was estimated to be approximately $26 \%$ using off axis injection with shared oscillations but with one obstacle still present in the machine. In this same configuration, a second measurement was taken after corrections were applied that gave $\Delta \beta / \beta_{h} \simeq 1.5 \%$ and $\Delta \beta / \beta_{v} \simeq 5.8 \%$ and $\Delta \eta_{h} \simeq 3.0 \mathrm{~mm}$ and $\Delta \eta_{v} \simeq 1.4 \mathrm{~mm}$. The injection efficiency after correction and once the last obstacle was removed improved to $60 \%$. The present situation, corresponding to the yellow curves, is the result of several iterations that converged to $\Delta \beta / \beta_{h} \simeq \Delta \beta / \beta_{v} \simeq 1.5 \%, \Delta \eta_{h} \simeq 1 \mathrm{~mm}$, and $\Delta \eta_{v} \simeq 1.0 \mathrm{~mm}$. A transfer efficiency from the booster to the storage ring of approximately $80 \%$ is routinely achieved in these conditions.

Coupling and vertical dispersion correction were performed and applied in parallel to linear optics corrections using the same ORM measurements. All available skew quadrupoles (SH, SF, and SD in Fig. 2) were used for these corrections. Measured vertical emittances below $1 \mathrm{pm} \mathrm{rad}$ are systematically obtained after coupling corrections without any further tuning, however, these values should be taken with great care as they are at the limit of the resolution of the pinhole cameras. In USM operation, the vertical emittance is stabilized at $10 \mathrm{pm}$ rad using a feedback loop injecting white noise in the vertical plane and the horizontal equilibrium emittance fluctuates between 120 and $130 \mathrm{pm}$ rad due to the ID gaps movements. This vertical emittance value was determined experimentally in collaboration with beam line experts that observed only 


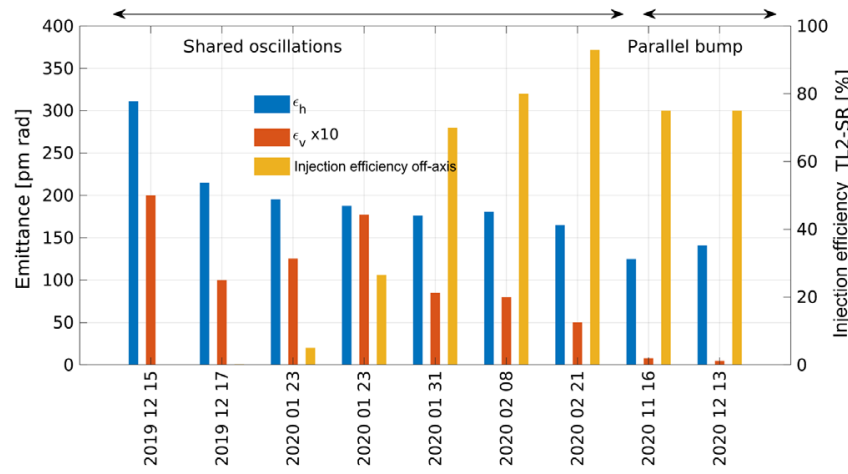

FIG. 13. Measured horizontal and vertical emittances and injection efficiency (off axis with shared stored-injected beam oscillations) during the first months of commissioning.

marginal improvement below this value. Small drifts or variations related to gap movements or orbit fluctuations are mitigated by the emittance feedback. Figure 13 shows the evolution of emittances and injection efficiency during the first months of commissioning.

\section{Integration of bending magnet beam lines}

The initial commissioning of the HMBA lattice was performed without the BM beam lines installed to facilitate the optics commissioning. $16 \mathrm{BM}$ beam lines were installed gradually toward the end of the machine commissioning period and during the beam lines commissioning period $[29,46]$. The machine now operates with all BM beam lines installed consisting of eight SB, seven $2 \mathrm{PW}$, and one $3 \mathrm{PW}$. While the installation of the $3 \mathrm{PW}$ is transparent for the lattice, the $2 \mathrm{PW}$ and $\mathrm{SB}$ require the adjustment of adjacent magnets to compensate for its impact on dispersion and orbit. The $2 \mathrm{PW}$ is compensated by rotating the quadrupole downstream of the source by 2 mrad around the vertical axis while the SB requires translation of all three DQs and two QF magnets and rematching of the cell to correct dispersion. These modifications were integrated by defining a new alignment reference. There was sufficient space between the magnets poles and the vacuum chamber to displace the magnets while keeping the chamber in place. On the first attempt, one 2PW and one SB were installed simultaneously. The magnet strength variations predicted from the model represented only a small increment. They were directly applied on top of existing corrections. Following this intervention, no first-turns steering was necessary as stored beam and accumulation were immediately possible without any correction.

For the specific case of the SB, further validation of the successful realignment is provided by closed orbit measurements: since the movements were applied to magnets only, large BPM to quadrupoles offsets were expected. This is shown in Fig. 14 where the expected and measured closed orbit variations are shown. The measured values are within $50 \mu \mathrm{m}$ from the expected values. A flat orbit

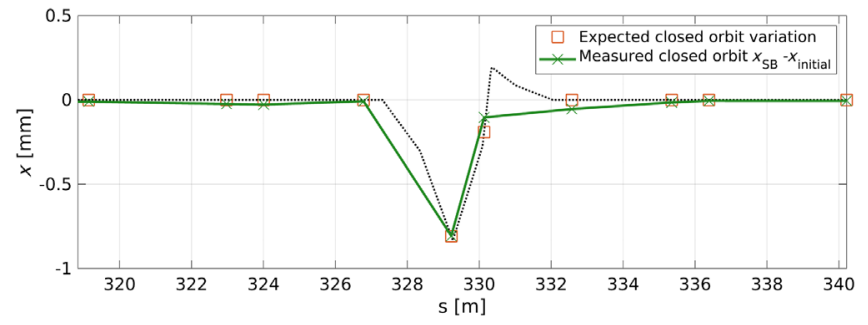

FIG. 14. Expected and measured closed orbit variation after installation of a short bend source. The measured values are derived from quadrupole to BPM offset measurements.

measurement is recovered when applying the measured BPM offsets. Following this successful experiment, up to 6 BM beam line sources were simultaneously installed over a single shutdown period without any difficulties in restarting the accelerators. The $\beta$-beating after a single SB installation is almost invisible, measurements following the installation gave $\Delta \beta / \beta_{h}=1.1 \pm 1 \%, \Delta \beta / \beta_{v}=1.3 \pm 1 \%$, $\Delta \eta_{h}=1.2 \pm 0.1 \mathrm{~mm}$, and $\Delta \eta_{v}=1.9 \pm 0.1 \mathrm{~mm}$ that is consistent with a fully corrected machine with potentially some slight increase in dispersion mismatch. The measured horizontal emittance with all BM beam lines installed is $130 \pm 20 \mathrm{pm} \mathrm{rad}$ which is consistent with model predictions of $140 \mathrm{pm}$ rad.

\section{Final corrections strengths}

Table III presents the correction strengths expected from simulations compared to the present storage ring corrections. Both dipole and quadrupole corrections needed to achieve the results from Table IV are lower than model prediction. This clearly reflects the outstanding work done during the engineering, construction, and installation of the ESRF-EBS storage ring.

In particular, using these correction strengths and the measured closed orbit and assuming alignment errors in quadrupoles and DQs only, it is possible to estimate the rms alignment errors throughout the ring. Although this model is certainly not capable of providing an accurate value, a validity range can be estimated. The results of these simulations are shown in Fig. 15. The blue and red areas are defined by the central values and standard deviations of simulations results performed with 20 seeds. The intercept with the measured values define the validity range. It is seen that the alignment of the storage ring ranges from 22 to $42 \mu \mathrm{m}$ in the horizontal plane and from 22 to $54 \mu \mathrm{m}$ in the

TABLE III. Final storage ring orbit and linear optics rms correction strength and comparison with simulations.

\begin{tabular}{lccc}
\hline \hline & Units & Simulations & Measured \\
\hline $\operatorname{rms} \theta_{h}$ & $\mu \mathrm{rad}$ & 160 & 65 \\
$\operatorname{rms} \theta_{v}$ & $\mu \mathrm{rad}$ & 120 & 30 \\
$\operatorname{rms~} \Delta K_{\text {quad }} / K_{\text {quad, } 0}$ & $\%$ & 0.26 & 0.2 \\
\hline \hline
\end{tabular}


TABLE IV. Design [29] and delivered parameters in USM condition and uniform mode. The design lifetimes were rescaled for $10 \mathrm{pm}$ rad vertical emittance for comparison purpose.

\begin{tabular}{lccc}
\hline \hline & Units & Design & Delivered \\
\hline$I_{e^{-}}$ & $\mathrm{mA}$ & 200 & 200 \\
Injection efficiency & $\%$ & $>90$ & 80 \\
Vacuum LT & $\mathrm{h}$ & 300 & $122 \pm 13$ \\
Touschek LT & $\mathrm{h}$ & $28 \pm 2$ & $41 \pm 5$ \\
USM LT & $\mathrm{h}$ & 23 & 25 \\
$\epsilon_{h}$ & $\mathrm{pm} \mathrm{rad}$ & 140 & $<130 \pm 20$ \\
$\epsilon_{v}$ & $\mathrm{pm} \mathrm{rad}$ & 10 & $10 \pm 1$ \\
rms orbit (x,y) & $\mu \mathrm{m}$ & 140,80 & 50,55 \\
Orbit stability (@20 Hz) & $\sigma$ & 0.05 & $<0.01$ \\
\hline \hline
\end{tabular}

vertical plane. This is much better than the tolerances of $70 \mu \mathrm{m}$ in both planes used to compute the design parameters in Table IV. As opposed to green field machines, the ESRF-EBS was aligned on the old machine to preserve the beam lines alignment. The alignment errors in the tunnel are defined as the deviations with respect to this nonzero reference. The estimated alignment error and uncertainty with respect to this reference is $53 \pm 40 \mu \mathrm{m}$ in the horizontal plane and $30 \pm 50 \mu \mathrm{m}$ in the vertical plane [47]. Although the errors themselves are relatively small, there are comparatively large uncertainties due to errors that potentially accumulate throughout the alignment process from the magnet fiducialization to the alignment on the girder, transport, and installation in the tunnel [48]. These estimates are consistent with the simulation results within error bars. Furthermore, it was shown that injection was possible without any need for vertical orbit correction, while horizontal corrections were needed only to accumulate. Finally, 26 out of the 27 ID beam lines could see light on the very first trial to open front-end without any need for adjustments. These two observations confirm the excellent machine alignment and validate the strategy of aligning the machine onto the reference of the previous machine rather than on a flat ideal model [29]. A remarkable feature of the HMBA lattice is that lattice corrections are applied only on quadrupole magnets located in the direct vicinity of sextupoles. This shows that optics errors are for the most part dominated by the feed-down effects in sextupole magnets driven by nonzero closed orbit at their location, as expected from the model. A $\beta$-beating level consistent with expectations could be achieved with lower than expected correction strengths. This is a direct consequence of the low-closed orbit and the accurate fiducialization and calibration of the magnets and PS. This localized correction scheme combined with the intrinsic resonance compensation of the lattice provides excellent stability of the corrections and only minor adjustments related to lifetime optimization with sextupoles, octupoles, and skew quadrupoles were required throughout the commissioning and during the first USM runs.
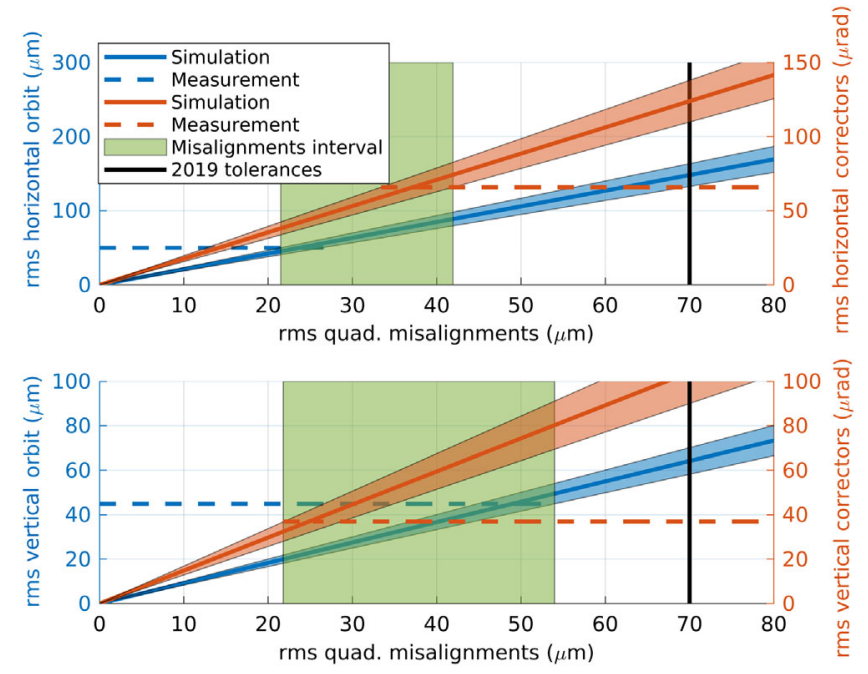

FIG. 15. Estimated SR alignment using 20 seeds of quadrupole and DQ displacement in simulations. The blue area represents the simulated rms orbit after correction and the red area the associated rms correctors strength. The dashed lines correspond to measured values and the green area the estimated misalignment range. The black line refers to the tolerances used in simulations.

\section{DELIVERING USM BEAM CONDITIONS}

The initial commissioning removed all physical obstacles and substantially increase the machine acceptance. The final obstacle, that represented the last major difficulty on the way to deliver operating conditions compatible with the start of beam lines commissioning, was removed 2 months after the start of commissioning. After that, the dispersive orbit used to avoid this obstacle could be removed and the TL2-SR transfer efficiency jumped from $17 \pm 5 \%$ to more than $30 \pm 5 \%$ to eventually reach $95 \pm 5 \%$ with shared oscillations a few days later. Efficient current ramp-up, conditioning of the vacuum chambers and the rf cavities to increasing beam loading $[49,50]$ allowed to achieve the nominal current of $200 \mathrm{~mA}$ before the end of the beam commissioning period.

Figure 16 shows the evolution of lifetime, average pressure, and total beam current as a function of the integrated beam current during the first year of operation. While the vacuum is still conditioning, the machine is now routinely operated with a lifetime greater than $20 \mathrm{~h}$ at the nominal current of $200 \mathrm{~mA}$. Periods with lower current after the initial commissioning period correspond to degraded running conditions related to sanitary restrictions or operation mode with lower current. These excellent lifetime performance were obtained by adjusting a large set of parameters, such as tunes, rf voltage or skew quadrupoles, sextupoles, and octupoles strengths. These optimizations took place on a regular basis during the vacuum conditioning and significant changes were applied in some case as the beam and vacuum conditions were improving. 


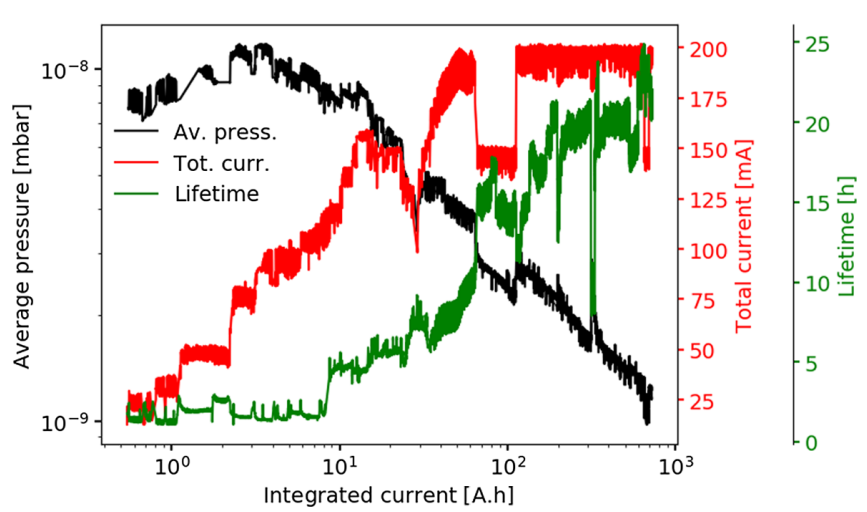

FIG. 16. Average pressure, lifetime, and total current evolution as a function of integrated current during the first year of operation.

These frequent adjustments proved to be crucial to speedup the current ramp-up.

During the vacuum conditioning, even though all physical obstacles were removed, unexpectedly low lifetime was observed at nominal current. This performance degradation was associated straight sections low gap chambers $(8 \mathrm{~mm}$ vertical aperture). One chamber was defective, causing a local vacuum degradation, excess of losses and high radiation levels. It had to be replaced leading to a significant improvement in overall performance with a reduction of approximately a factor 2 in emittance combined with a factor 2 increase in lifetime. This is seen on Fig. 16 at approximately $10 \mathrm{Ah}$ integrated current. Following this intervention, several chambers had to be realigned to minimize the losses in the straight sections. In these cases, vertical parallel bump scans were performed to determine the optimum position. Displacements were of the order of a few $100 \mu \mathrm{m}$. Finally, to speed-up the conditioning process, slow orbit fluctuations were applied during the conditioning runs to expose parts of the vacuum chambers that are normally not irradiated by the beam circulating on axis to $\mathrm{x}$ rays. These fluctuations were generated by two correctors separated in phase by $\pi / 2$ in order to scan the phase of the perturbation while maintaining the rms orbit constant. It should be noted that the vertical emittance was increased to $20 \mathrm{pm}$ rad to increase the lifetime during the first part of the vacuum conditioning and only later was it decreased to $10 \mathrm{pm}$ rad.

Figure 17 shows a fit of the lifetime as a function of the vertical emittance, set with white noise excitation, from which the Touschek and vacuum lifetimes can be extrapolated. These measurements were taken after approximately 1.5 years of operation. The vacuum lifetime remains low compared to the design goal of $300 \mathrm{~h}$ and contributes to the overall lifetime. This confirms that the vacuum is not yet fully conditioned. The Touschek lifetime is estimated at $30 \pm 3 \mathrm{~h}$ at $5 \mathrm{pm}$ rad. The average value for lattices generated using 10 seeds of errors is approximately $22 \pm 1 \mathrm{~h}$ [46] and the ideal lattice after full optimizations

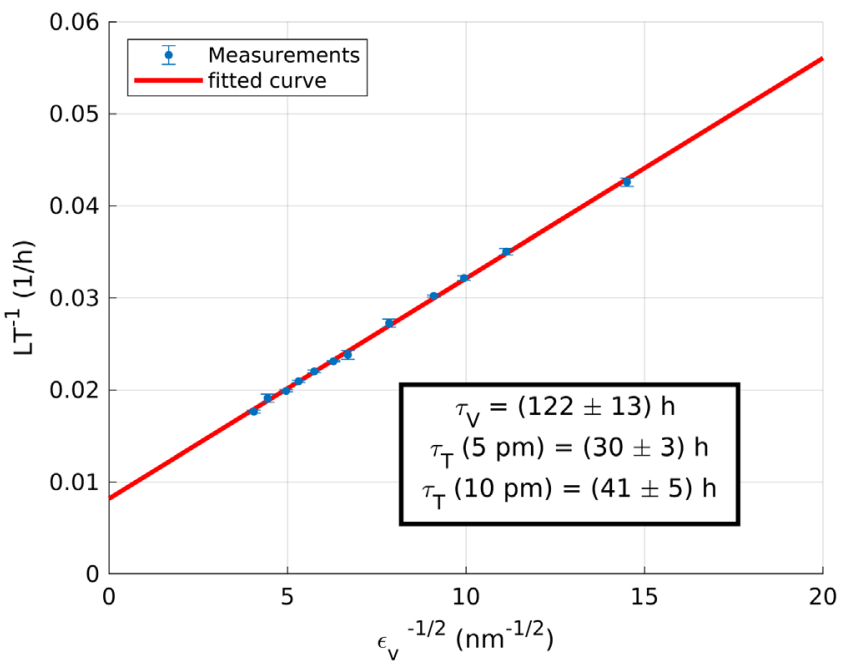

FIG. 17. Vacuum $\left(\tau_{V}\right)$ and Touschek lifetimes $\left(\tau_{T}\right)$ estimates after 1.5 years of operation. LT is the total measured lifetime and $\epsilon_{v}$ the measured vertical emittance.

of sextupoles and octupoles gives $42 \mathrm{~h}$ [28]. Several reasons can explain why the measured lifetime exceeds realistic model with errors predictions: (i) The measured alignment errors are better than the specifications used in model calculations and consequently the closed orbit is smaller, see Table IV, (ii) A single set of sextupoles and octupoles based on the average of the ten seeds of errors was used in the model, and (iii) Online lifetime optimizations took full advantage of the individual PS: during the early commissioning individual magnets were used, and later, combinations derived from singular vectors of the orbit response matrix as well as on-energy and off-energy optics response matrices. Similar optimizations during the design phase only considered few families: one octupole family and nine sextupole families, six for injection and three for the standard cells.

With all these optimizations integrated in the operation settings, lifetime tuning for USM now relies on online optimizations that consist in minimizing the losses measured by the BLD system by scanning selected combinations of sextupoles, octupoles, and skew quadrupoles magnets. The implementation of individual PS for all these magnets as part of the ESRF storage ring upgrade therefore proves to be essential for performance optimization. These are performed on a regular basis and after each shutdown period to maintain a lifetime $>20 \mathrm{~h}$ at $200 \mathrm{~mA}$.

With a fully optimized storage ring, the machine operates routinely with ID gaps closed at an injection efficiency of approximately $80 \%$, below the design goal of $90 \%$. Even though the simulations did not integrate vertical aperture restrictions or imperfections such as energy mismatch or vertical injection oscillations and assumed a low-emittance optics in the booster that is not yet implemented, the implementation of emittance exchange at the booster extraction should have compensated for this loss. 


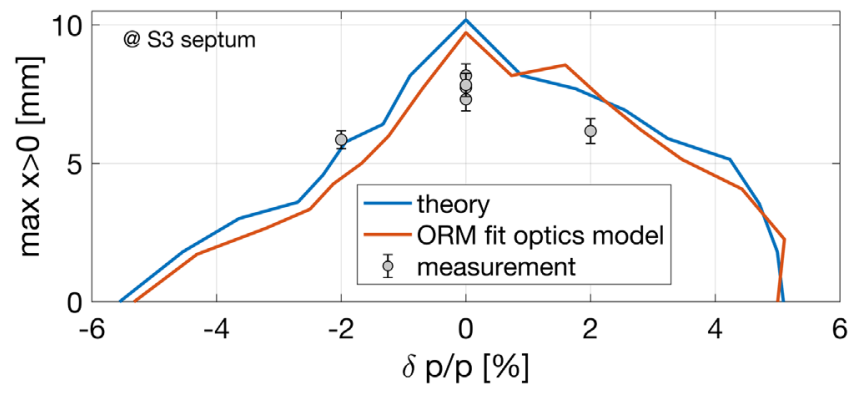

FIG. 18. Simulated and measured dynamic aperture. Blue corresponds to the perfect lattice and red to the model fitted from ORM measurements.

Figure 18 shows the measured and model DA. The ideal lattice and the fitted model are shown in blue and red, respectively. The measured on-momentum DA is smaller than model prediction by 1 to $2 \mathrm{~mm}$ which could explain lower than expected injection efficiency. Improvements can therefore be expected with further optimizations of the nonlinear optics. This observation combined with larger than expected lifetime could indicate that excessive weight was put on lifetime in the optimization process and further studies are required to find the proper balance between these two performance parameters.

The ESRF serves a large number of modes [2] to the beam lines users with bunch current as high as $10 \mathrm{~mA}$. Single bunch collective effects have been evaluated and are for the most part consistent with the impedance model predictions [51]. They do not limit the single bunch current as the maximum design value of $10 \mathrm{~mA}$ could easily be achieved with either increased chromaticity or making use of the bunch-by-bunch feedback. However, beam induced heating on the ceramic chambers generates excessive mechanical stress that the present design cannot sustain. The procurement of new more robust ceramic chambers was launched to overcome this issue that presently limits the single bunch current to $4 \mathrm{~mA}$ and the total current of the few bunch modes from $1 / 3$ to $3 / 4$ of the design value.

\section{SUMMARY AND OUTLOOK}

After one year of operation including 3 months of machine commissioning and 5 months of beam line commissioning, the ESRF-EBS accelerator facility resumed USM operation on August 5, 2020 as planned and is now running with beam conditions matching, or even exceeding in some aspects, the design expectations.

Table IV compares the design and delivered beam parameters. The results are excellent and matching design goals in all aspects except for the injection efficiency. The horizontal emittance fluctuates between 120 and $130 \mathrm{pm}$ rad depending on insertion devices gap settings and the vertical emittance is fixed at $10 \mathrm{pm}$ rad to increase the lifetime as for the moment no significant improvement in performance was observed by the beam lines going to lower values. This value will be reevaluated in the future. The beam stability is measured with all feedbacks running on a short timescale, long-term stability is being evaluated. Three lifetime values are quoted in this table: the vacuum lifetime evaluated with vertical emittance scans, the Touschek lifetime rescaled to $10 \mathrm{pm}$ for the design and measured with all gaps and collimators opened on a fully optimized machine and the USM lifetime which corresponds to the average lifetime delivered at $200 \mathrm{~mA}$ over one week of uniform mode (992 bunches with $0.02 \mathrm{~mA}$ per bunch). This value is reduced by approximately $5-10 \%$ by the collimation systems [52] used to localize Touschek losses in shielded areas. The design value assumed a pessimistic $10 \%$ reduction. With a fully optimized storage ring, the machine operates routinely with ID gaps closed at an injection efficiency of approximately $80 \%$ and a total lifetime of approximately $25 \mathrm{~h}$ in uniform mode. The machine availability and mean time between failures are approaching the levels of the previous machine with $96.1 \%$ and $46 \mathrm{~h}$ for the 2 runs of 2020 and $97.9 \%$ and $103.5 \mathrm{~h}$ for the first 2 runs of 2021 [53].

The ESRF HMBA lattice was designed to match strict constraints imposed by the in-place infrastructure such as the tunnel and the beam lines. The performance and results presented in the above sections exceed by far our expectations and showcases the original design of this lattice. However, room is left for further improvements based on our growing understanding of the lattice. Further reduction of the photon source size by redistributing the damping partition number and reduction the $\beta$-functions in straight while increasing the acceptance of the machine with better matching of the optics off-energy is within reach and studies are ongoing in this direction.

\section{ACKNOWLEDGMENTS}

The authors would like to thank all members of the ASD division for their help and fruitful discussions over the whole commissioning period. In particular, a special thank goes to the operation group for their support during lengthy and sometimes very late control room shifts, to the diagnostics group for their readiness and reactivity in providing us with all these accurate and essential data and finally to the accelerator control unit for all the tools developed before, during, and after the commissioning that really made our lives much easier.

[1] Science and technology programme 2008-2017 (Purple book), ESRF, 2007.

[2] ESRF upgrade programme phase II (Orange book), ESRF, 2014.

[3] D. Einfeld, J. Schaper, and M. Plesko, Design of a diffraction limited light source (DIFL), in Proceedings of PAC'95 (1995), Vol. 1, pp. 177-179. 
[4] H. Tarawneh, M. Eriksson, L. J. Lindgren, and B. Anderberg, MAX-IV lattice, dynamic properties and magnet system, Nucl. Instrum. Methods Phys. Res., Sect. A 508, 480 (2003).

[5] M. Magnuson et al., MAX IV Conceptual Design Report (CDR), Lund, 2006.

[6] P. F. Tavares, E. Al-Dmour, . Andersson, F. Cullinan, B. N. Jensen, D. Olsson, D. K. Olsson, M. Sjstrm, H. Tarawneh, S. Thorina, and A. Vorozhtsova, Commissioning and firstyear operational results of the MAXIV $3 \mathrm{GeV}$ ring, J. Synchrotron Radiat. 25, 1291 (2018).

[7] The ESRF foundation report, Red Book, ESRF, 1992.

[8] A. W. Chao and M. Tigner, Handbook of Accelerator Physics and Engineering (World Scientific, Singapore, 1999).

[9] L. Farvacque, N. Carmignani, J. Chavanne, A. Franchi, G. Le Bec, S. Liuzzo, B. Nash, T. Perron, and P. Raimondi, A low-emittance lattice for the ESRF, in Proceedings of IPAC'13 (2013), MOPEA008, pp. 79-81.

[10] S. White, J. Chavanne, M. Dubrulle, G. Le Bec, E. Plouviez, P. Raimondi, and B. Roche, Damping of injection perturbations at the European Synchrotron Radiation Facility, Phys. Rev. Accel. Beams 22, 032803 (2019).

[11] P.Elleaume and A. Ropert, The ultimate hard X-ray storage ring based light source, Nucl. Instrum. Methods Phys. Res., Sect. A 500, 18 (2003).

[12] Y. Cai, K. Bane, R. Hettel, Y. Nosochkov, M. Wang, and M. Borland, Ultimate storage ring based on fourth-order geometric achromats, Phys. Rev. ST Accel. Beams 15, 054002 (2012).

[13] V. Sajaev, Commissioning simulations for the Argonne Advanced Photon Source upgrade lattice, Phys. Rev. Accel. Beams 22, 040102 (2019).

[14] C. Steier et al., Completion of the brightness upgrade of the ALS, J. Phys. Conf. Ser. 493, 012030 (2014).

[15] J. C. Biasci et al., A low emittance lattice for the ESRF, Synchrotron Radiat. News 27, 8 (2014).

[16] S. Papadopoulou, F. Antoniou, and Y. Papaphilippou, Emittance reduction with variable bending magnet strengths: Analytical optics considerations and application to the Compact Linear Collider damping ring design, Phys. Rev. Accel. Beams 22, 091601, 2019.

[17] R. Nagaoka and A. Wrulich, Emittance minimisation with longitudinal dipole field variation, Nucl. Inst. Meth. AB Nucl. Instrum. Methods Phys. Res., Sect. A 575, 292 (2007).

[18] H. Wiedemann, Particle Accelerator Physics (Springer, New York, 2007).

[19] M. Bona et al., SuperB: A high-luminosity asymmetric $e^{+} e^{-}$super flavor factory, Conceptual Design Report, INFN/AE - 07/2, SLAC-R-856, LAL 07-15, 2007.

[20] P. Raimondi and A. Seryi, A Novel Final Focus Design for Future Linear Colliders, Phys. Rev. Lett. 86, 3779 (2001).

[21] K. Oide, M. Aiba, S. Aumon, M. Benedikt, A. Blondel, A. Bogomyagkov et al., Design of beam optics for the future circular collider e+e- collider rings, Phys. Rev. Accel. Beams 19, 111005 (2016).

[22] S. Liuzzo, N. Carmignani, J. Chavanne, L. Farvacque, G. Le Bec, B. Nash, P. Raimondi, R. Versteegen, and S. White, Updates on lattice modeling and tuning for the ESRF-EBS lattice, in Proceedings of IPAC'16 (2016), WEPOW005.
[23] S. M. Liuzzo et al., Influence of errors in the ESRF upgrade lattice, in Proceedings of IPAC'15 (2015), pp. 1426-1429, TUPWA014.

[24] C. Benabderrahmane, J. C. Biasci, J. F. Bouteille, J. Chavanne, L. Farvacque, L. Goirand, G. Le Bec, S. M. Luizzo, P. Raimondi, and F. Villar, Magnets for the ESRF-EBS project, in Proceedings of IPAC'16 (2016), pp. 1096-1099, TUPMB001.

[25] G. Le Bec, S. Liuzzo, F. Villar, P. Raimondi, and J. Chavanne, Single sided dipole-quadrupole magnet for the Extremely Brilliant Source storage ring at the European Synchrotron Radiation Facility, Phys. Rev. Accel. Beams 22, 102402 (2019).

[26] G. Le Bec, J. Chavanne, F. Villar, C. Benabderrahmane, S. Liuzzo, J.-F. Bouteille, L. Goirand, L. Farvacque, J.-C. Biasci, and P. Raimondi, Magnets for the ESRF diffractionlimited Light Source Project, IEEE Trans. Appl. Supercond. 26, 1 (2016).

[27] N. Carmignani, Touschek Lifetime Studies and Optimization of the European Synchrotron Radiation Facility (Springer, New York, 2016).

[28] N. Carmignani, L. Farvacque, S. M. Liuzzo, B. Nash, T. Perron, P. Raimondi, R. Versteegen, and S. White, Linear and Nonlinear Optimizations for the ESRF Upgrade Lattice, in Proceedings of IPAC'15 (2015), pp. 1422-1425, TUPWA013.

[29] S. M. Liuzzo, N. Carmignani, J. Chavanne, L. Farvacque, B. Nash, and P. Raimondi, Optics adaptations for bending magnet beam lines at ESRF: Short bend, 2-pole Wiggler, 3-pole Wiggler, in Proceedings of IPAC'17 (2017), pp. 666-669 MOPIK062.

[30] J. Chavanne et al., Magnet developments for the ESRFEBS, presented at LER'16, 2016.

[31] N. Carmignani et al., Operation improvements and emittance reduction of the ESRF booster, in Proceedings of IPAC'18 (2018), pp. 4077-4080, THPMF017.

[32] N. Carmignani, L. R. Carver, S. M. Liuzzo, T. Perron, and S. White, Operation of the ESRF Booster with the new EBS Storage Ring, in Proceedings of IPAC'21 (2021), MOPAB051.

[33] S. Liuzzo, N. Carmignani, A. Franchi, T. Perron, K. B. Scheidt, E. Taurel, L. Torino, and S. White, Preparation of the EBS Beam Commissioning, J. Phys. Conf. Ser. 1350, 012022 (2019).

[34] G. Le Bec, J. Chavanne, S. Liuzzo, and S. White, Crosstalks between storage ring magnets at the Extremely Brilliant Source storage ring at the European Synchrotron Radiation Facility, Phys. Rev. ST Accel. Beams 24, 072401 (2021).

[35] S. M. Liuzzo, N. Carmignani, L. R. Carver, L. Farvacque, T. Perron, P. Raimondi, and S. White, HMBA optics correction experience at ESRF, in Proceedins of IPAC'21 (2021), TUPAB048.

[36] L. Torino, N. Benoist, F. Ewald, E. Plouviez, J. Poitou, B. Roche, K. B. Scheidt, F. Taoutaou, and F. Uberto, Overview on the diagnostics for EBS-ESRF, in Proceedings of IBIC'19 (2019), pp. 9-13, MOAO03.

[37] A. Nosych, U. Iriso, and J. Olle, Electrostatic Finiteelement Code to Study Geometrical Nonlinear Effects of BPMs in 2D, in Proceedings of IBIC'15 (2015), pp. 418-422, TUPB047. 
[38] M. Minty and F. Zimmermann, Measurement and control of charged particle beams (Springer, New York, 2003).

[39] Z. Marti, G. Benedetti, U. Iriso, and A. Franchi, Fast beambased alignment using ac excitations, Phys. Rev. Accel. Beams 23, 012802 (2020).

[40] E. Plouviez and F. Uberto, The orbit correction scheme of the new EBS of the ESRF, in Proceedings of IBIC'16 (2016), pp. 51-54, MOPG09.

[41] G. Le Bec, Magnetic measurements and fiducialisation of the ESRF-EBS magnets, in Proceedings of IPAC20 (2020).

[42] G. Le Bec, J. Chavanne, and C. Penel, Stretched wire measurement of multipole accelerator magnets, Phys. Rev. ST Accel. Beams 15, 022401 (2012).

[43] A. Franchi, L. Farvacque, J. Chavanne, F. Ewald, B. Nash, K. B. Scheidt, and R. Tomas, Vertical emittance reduction and preservation in electron storage rings via resonance driving terms correction, Phys. Rev. ST Accel. Beams 14, 034002 (2011).

[44] R. Tomas, M. Aiba, A. Franchi, and U. Iriso, Review of linear optics measurement and correction for charged particle accelerators, Phys. Rev. Accel. Beams 20, 054801 (2017).

[45] L. Malina, J. Coello de Portugal, A. Langner, T. Persson, P. K. Skowronski, R. Tomas, L. Farvacque, and A. Franchi, Comparison of optics measurement methods in ESRF, in Proceedings of IPAC'16 (2016), pp. 3343-3346, THPMB045.
[46] S. M. Liuzzo, N. Carmignani, J. Chavanne, L. Farvacque, T. Perron, P. Raimondi, and S. White, ESRF-EBS lattice model with canted beamlines, J. Phys. Conf. Ser. 1067, 032006 (2018).

[47] D. Martin, ESRF-EBS Alignment, Presented at the Virtual Mini-Workshop on Girders and Alignment, 2021.

[48] The Guide to the Expression of Uncertainty in Measurement, JCGM, 1993.

[49] J. Jacob, P. Borowiec, A. D'Elia, G. Gautier, and V. Serriere, ESRF-EBS $352.37 \mathrm{MHz}$ radio frequency system, in Proceedings of IPAC'21 (2021), MOPAB 108.

[50] A. D'Elia, J. Jacob, V. Serriere, and X. Zhu, ESRF-EBS $352 \mathrm{MHz}$ HOM damped RF cavities, in Proceedings of IPAC'21 (2021), MOPAB332.

[51] L. Carver, E. Buratin, N. Carmignani, F. Ewald, L. Hoummi, S. Liuzzo, T. Perron, B. Roche, and S. White, Single bunch collective effects in the EBS storage ring, in Proceedings of IPAC'21 (2021), MOPAB117.

[52] R.Versteegen, P. Berkvens, N. Carmignani, L. Farvacque, S. M. Liuzzo, B. Nash, T. Perron, P. Raimondi, and S. White, Collimation scheme for the ESRF upgrade, in Proceedings of IPAC'15 (2015), pp. 1434-1437, TUPWA017.

[53] J. L. Revol et al., ESRF-EBS: Implementation, performance and restart of user operation, in Proceedings of IPAC'21 (2021), THPAB074. 